\title{
Decentralized Nonlinear Control Strategies for Disturbance Rejection in Winding Systems
}

\author{
Fouad Mokhtari \\ Pierre Sicard \\ Groupe de Recherche en Électronique Industrielle (GREI) \\ Université du Québec à Trois-Rivières \\ C.P.500, Trois-Rivières (Québec), Canada G9A-5H7 \\ Email: Fouad.Mokhtari@uqtr.ca,Pierre.Sicard@uqtr.ca
}

\author{
Abdeldjbar Hazzab \\ Laboratoire de Commande, Analyse et Optimisation \\ Université de Bechar \\ B.P. 417, Bechar, 08000, Algeria \\ Email: a_hazzab@yahoo.fr
}

\begin{abstract}
In winding and web transport systems, which are complex and large scale interconnected dynamics systems with a demand for increasingly high level of performance, the objective is to ensure quality processing and rewinding of the product. A major problem in controlling such systems is that disturbances propagate through the system and affect both velocity and tension loops because of the strong mechanical couplings. An active disturbance rejection control (ADRC) strategy and a nonlinear PI (NPI) controller are proposed and compared with PI control to improve the overall performance of the system, i.e. increasing dynamic tracking precision, reducing rise time for step input and furthermore improving robustness to the change of the web stiffness during the winding process. Simulation results of an industrial application show the effectiveness and disturbance rejection properties of the proposed controllers in coping with large dynamic variations commonly seen in web handling applications.
\end{abstract}

Index Terms-Winding systems, disturbance rejection, decentralized control, web tension control.

\section{INTRODUCTION}

Many types of materials are manufactured or processed in the form of a web or sheet (textile, paper, metal), which then couples the processing rolls and the associated motor drives. The drives are required to work in synchronism to ensure quality processing and rewinding of the product. Tension is a very important web manufacturing and process setting. Severe tension variations may result into rupture of the materiel during processing or degradation of product quality causing significant economic losses due to material loss and reduced production rate. Therefore, in order to minimize the potential for losses, the need arises to adequately control the tension within a predefined range in a moving web processing section.

The problem of tension control in web handling system is complex because the system dynamics is highly nonlinear and is function of many variables of the process that often vary in a wide range (changes and variations in the diameter and inertia of rolls, elasticity modulus and section of web). The unmodelled dynamics and uncertainties are considered as disturbances in the system. Disturbances affecting velocity propagate to the tension of the web, with complex dynamics due to the inherent flexibility of the web. All these, make the control problem challenging.

The increasing demand for quality and efficiency in industry has motivated many researchers and engineers alike to explore better methods for velocity and tension control. Several control strategies based on decentralized or centralized multivariable control sctructure have been suggested for winding and web transport systems in order to achieve the control objectives, such as : PID [1][2], loop shaping [3], gain scheduling [4], $\mu$-analysis [5], multivariable $H_{\infty}$ control [6]. It led also to the investigation for advanced control strategies including : fuzzy logic [7], neural networks [8], optimum control [9] and robust control [10][11].

The conventional PID control dominates industry, it is simple and easy to implement. Tuning of PID controllers is intuitive and is well accepted by practitioners. PIDs can at most achieve a compromise in performance in terms of system response speed and stability, and this approach becomes insufficient at the increasingly high web velocities demanded by the industry and with thin or fragile materials. Nonlinearities that appear at high velocities, disturbance rejection properties and robustness to some parameter variations must be accounted for by the controller.

In this paper, we propose and evaluate an active disturbance rejection control (ADRC) strategy and a nonlinear PI (NPI) controller for distributed control to reduce sensitivity to external disturbances in winding and web transport systems. The ADRC approach is known for its effectiveness in rejecting disturbances ([12][13] and references therein). In particular, it allows decoupling the control action by treating all unknown coupled dynamics as being a generalized term and it compensates them in real time using an extended state observer, without the necessity of an explicit model of disturbances and unknown dynamics. While, the improvement of NPI controller is achieved by the use of nonlinear gains [14], the combination of nonlinear terms can provide additional degrees of freedom to achieve a much improved system performance. The NPI controller can adjusts its gains in real time according to the tension error and the trend of the tension variations and it is able to reject the effect of time-varying and nonlinear behaviors in the winding process.

This paper is organized as follows. Section II presents the model of a five drive test winding system and section III, introduces the ADRC strategy and NPI controller for web transport systems. Simulation results and performance of the controllers are compared in section IV. Concluding remarks are presented in section $\mathrm{V}$. 


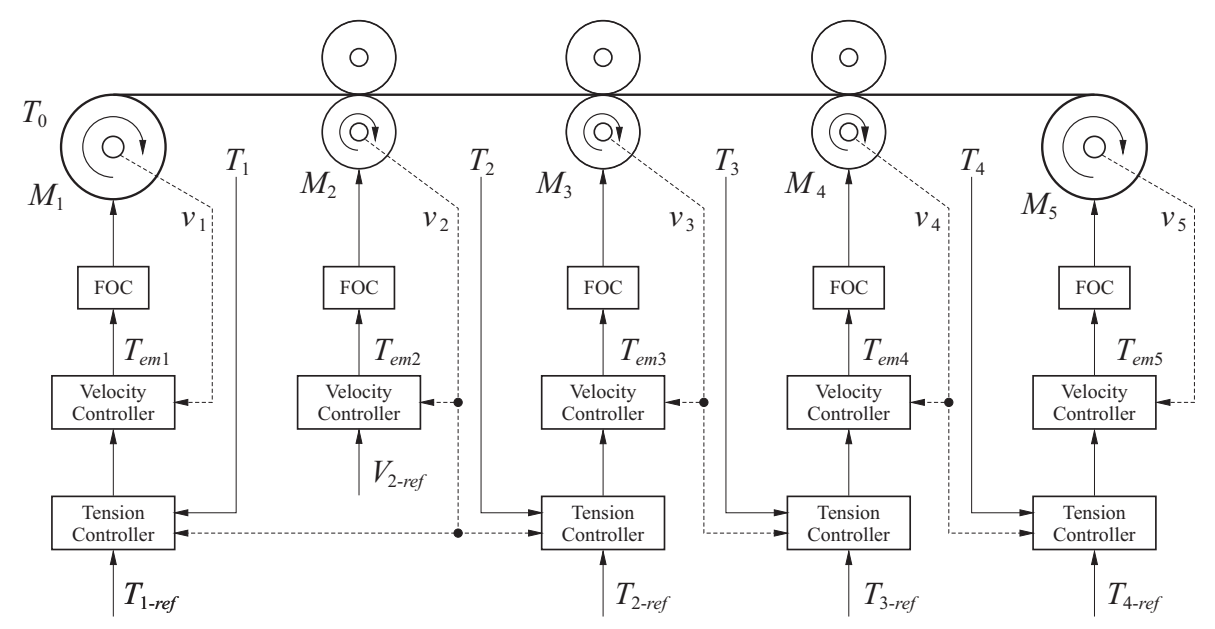

Fig. 1. Winding system.

\section{Test winding System Model}

Consider a simple winding system composed of five drives given in Fig.1. The unwind roll is driven by induction machine $M_{1}$. The winder roll is driven by induction machine $M_{5}$. Three tension rolls are driven by induction machines $M_{2}, M_{3}$ and $M_{4}$. Web velocity $v_{2}$ and tension in each web segment are controlled.

\section{- Hypothesis:}

i) The web velocity is equal to the roll surface linear velocity (slip is neglected).

ii) Variations of inertias and of radii are typically slow as compared to the dynamics of velocities and tensions and are neglected in this study.

A simplified model for the web tension between two consecutive rolls is obtained from simplified Hook's law [6][14].

$$
L_{k} \frac{d T_{k}}{d t}=E S \triangle v_{k+1}-v_{k} \triangle T_{k}
$$

The velocity $v_{k}$ of the $k$ th roll can be obtained by

$$
J_{k} \frac{d v_{k}}{d t}=r_{k}^{2} \triangle T_{k}-f_{k} v_{k}+r_{k} T_{e m_{k}}
$$

where $\triangle v_{k+1}=v_{k+1}-v_{k}$ and $\triangle T_{k}=T_{k}-T_{k-1} ; f_{k}$ and $J_{k}$ are respectively the viscous friction coefficient and the equivalent total inertia on the shaft of motor $k ; r_{k}$ is the radius of roll $k ; T_{e m_{k}}$ is the electromagnetic torque of motor drive $k ; T_{k}$ is the web tension between roll $k-1$ and roll $k ; E$ is the elasticity modulus of the web; $S$ its section and $L_{k}$ is the web length between rolls $k-1$ and $k$.

\section{IMPROVEMENT OF PERFORMANCE AND DISTURBANCE REJECTION}

For web transport systems with high level of performance, it is not suitable to use a centralized controller because of the large dimension of the model. Therefore, a solution to reduce the coupling between adjacent sub-systems is to use a decentralized control structure [15][16], or semi-centralized control,

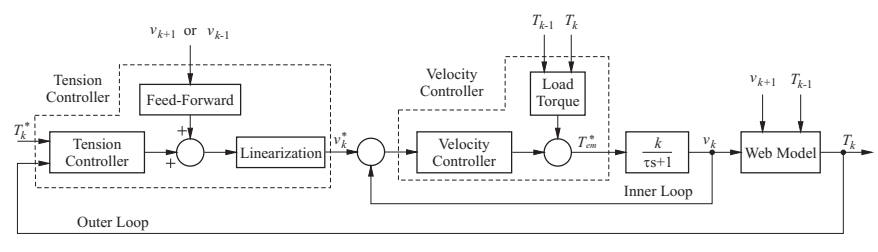

Fig. 2. Cascade control structure applied on the motors

i.e. decentralized control with overlapping and interactions [17] [18].

The objective is to control the tension in web segments and velocity of the traction roll to meet production and quality specifications. To achieve this objective, a cascade decentralized controller is applied since cascade and decentralized control systems depend more strongly on feedback rather than models as their source of information.

The decentralized control structure shown on Fig. 1 is considered, where a velocity controller is associated to each motor; the velocity references are set by the external tension control loop and by the desired web velocity. The tension dynamics is very sensitive to the velocity variations because of coupling. Nevertheless, the control of the internal velocity loop in the Fig.2 is the key for a good control of tension loop. If stability is assured, it remains to find a solution in order to improve the performance of the tension response to avoid oscillations due to the variations of the parameters in the winding system (Young modulus, inertia, ...) and to disturbance caused, for example, by the variation of the load torque and of tension and velocity references. The new structure of control should not improve only performance, but it should also be simple to design, to implement and to regulate. To achieve this, two controller strategies are proposed.

\section{A. Decentralized Nonlinear PI (NPI) Controller}

In order to improve the control quality, a nonlinear PI velocity controller can be constructed as shown in Fig.3 [14]. It is composed of a nonlinear PI combination in order to 


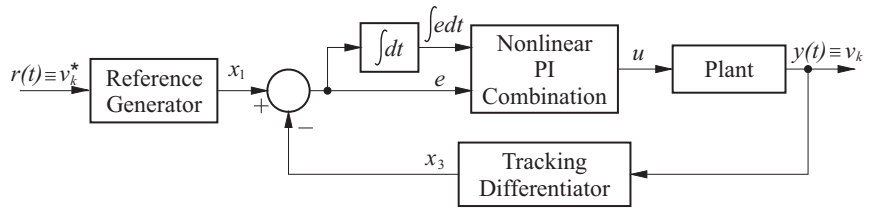

Fig. 3. Nonlinear PI controller

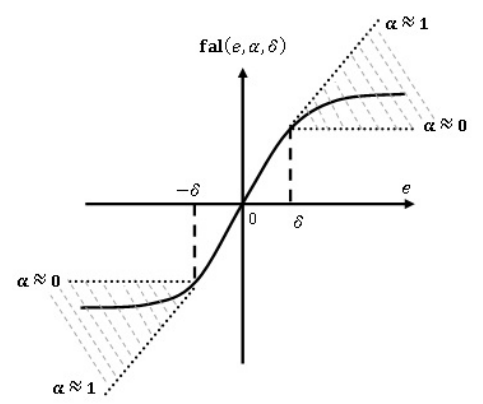

Fig. 4. $\mathbf{f a l}(e, \alpha, \delta))$ function characteristics.

produce the control action, a nonlinear reference generator and a tracking differentiator. The combination of nonlinear terms can provide additional degrees of freedom to achieve a much improved system performance. The reference generator in the forward path yields the high quality differential signal of reference and the tracking differentiator in the feedback path yields the feedback differential signal, both in the presence of measurement noise or rapidly changing disturbance signals.

The nonlinear PI (NPI) controller action is given by

$$
\begin{aligned}
& u=K_{p} \mathbf{f a l}\left(e, \alpha_{p}, \delta_{p}\right)+K_{i} \mathbf{f a l}\left(\int e d t, \alpha_{i}, \delta_{i}\right) \\
& \mathbf{f a l}(e, \alpha, \delta)= \begin{cases}|e|^{\alpha} \operatorname{sign}(e) & |e|>\delta, \delta>0 \\
\frac{e}{\delta^{1-\alpha}} & |e| \leq \delta\end{cases}
\end{aligned}
$$

where a nonlinear function fal $(e, \alpha, \delta)$ is given by (4) and (Fig.4); $K_{p}$ and $K_{i}$ are respectively proportional and integral gains of the NPI controller; $u$ is the control signal; parameters $\alpha_{p}$ and $\alpha_{i}$ are constant, empirically chosen in the range 0 to 1 . When $\alpha_{i}=\alpha_{p}=1$, the controller becomes a linear PI controller. Henceforth, design of a linear PI controller can be used for initial setting of $K_{p}$ and $K_{i}$ in (3). $\delta$ is a constant, which can be set empirically to a small value.

The equations for reference generator and tracking differentiator can be respectively expressed as

$$
\begin{aligned}
& \left\{\begin{array}{l}
\dot{x}_{1}=x_{2} \\
\dot{x}_{2}=-R_{1} \operatorname{sat}\left(x_{1}-r(t)+\frac{x_{2}\left|x_{2}\right|}{2 R_{1}}, \sigma\right)
\end{array}\right. \\
& \left\{\begin{array}{l}
\dot{x}_{3}=x_{4} \\
\dot{x}_{4}=-R_{2} \operatorname{sat}\left(x_{3}-y(t)+\frac{x_{4}\left|x_{4}\right|}{2 R_{2}}, \sigma\right)
\end{array}\right.
\end{aligned}
$$

where $\operatorname{sat}(A, \sigma)$ is a saturation function defined by

$$
\operatorname{sat}(A, \sigma)= \begin{cases}\operatorname{sign}(A), & |A| \geq \delta, \\ \frac{A}{\sigma}, & |A|<\delta\end{cases}
$$

$r(t)$ and $y(t)$ are respectively the reference and measured feedback signal of the plant; $R_{1}$ and $R_{2}$ are the filter's design parameters, which can be determined empirically; $\sigma$ is a small design parameter. The filter parameters determine the rapidity of the transients and the differential tracking performance: large value for $R_{1}$ and $R_{2}$ give a fast transient and improved tracking performance. Initial setting for $R_{1}$ and $R_{2}$ can be obtained from a linear approximation of (5) and (6) around an operation point.

\section{B. Decentralized Active Disturbance Rejection Control (ADRC) Strategy}

The method of active disturbance rejection control (ADRC) [12][13], because of its robustness and disturbance rejection capabilities, is notably suitable and useful for control of the tension in winding and web transport systems. ADRC is designed under the assumption of a high degree of model uncertainties and is inherently robust against plant variations. The ADRC strategy is not dependent on the detailed model of the system, but on its inputs and outputs. The tension dynamics of web transport systems are partially decoupled by the exchange of velocity information among the drives. However, the rolls dynamics are strongly coupled by the tension terms in (1) and (2), and parameter uncertainties and unmodeled dynamics due to modeling approximations cause residual couplings and disturbances in the system. We propose to model the coupling tension dynamics as disturbances and to replace the velocity loops in Fig. 1 by the ADRC strategy of Fig. 5 to decouple the tension dynamics from the velocity loops.

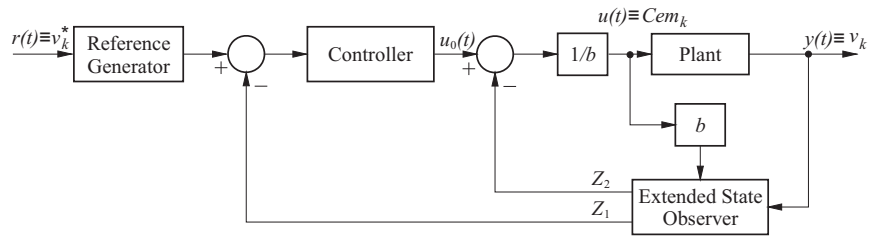

Fig. 5. Active disturbance rejection control applied to the velocity loop.

The architecture of ADRC control structure illustrated in Fig. 5 is composed of an observer, a reference generator and PI velocity control. The extended state observer produces the estimated value of the extended state that includes the total disturbance, which is composed of the external disturbance and unmodelled dynamics, whereas the reference generator produces the approximate value of time derivatives of the reference input. In [19], it is shown analytically how to use singular perturbation theory to obtain a sufficient condition for exponential stability of the system in closed loop with active disturbance rejection control (ADRC) for a nonlinear system with time varying parameters.

To apply a ADRC to velocity control loops, the velocity equations (2) of the winding system are rewritten as

$$
\dot{v}_{t}=f(\cdot)+b u
$$



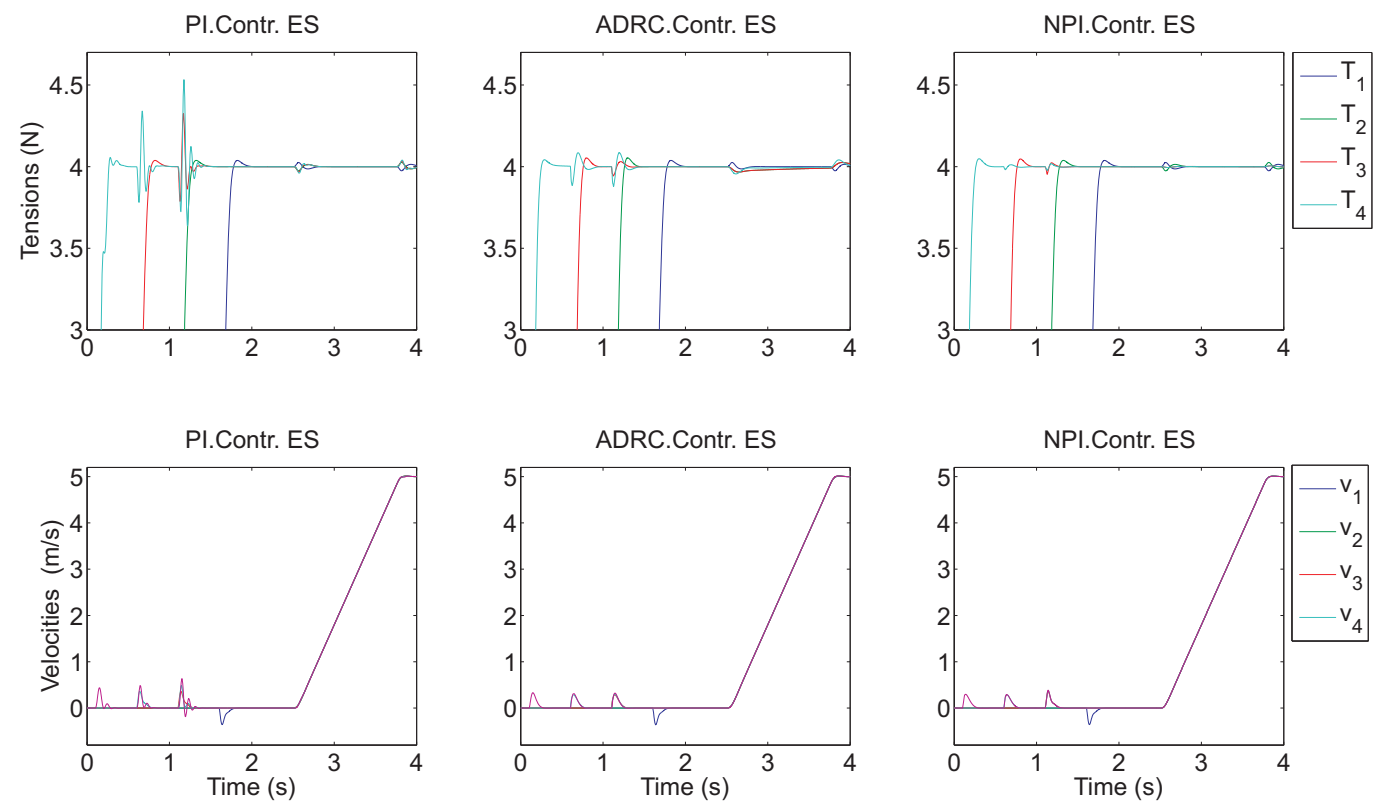

Fig. 6. Tensions and velocities responses for standard PI, NPI and ADRC controllers with exact parameters.

with

$$
\begin{aligned}
& f(\cdot)=\left(\begin{array}{c}
\frac{1}{J_{1}}\left[r_{1}^{2} T_{1}-f_{1} v_{1}\right] \\
\frac{1}{J_{2}}\left[r_{2}^{2}\left(T_{2}-T_{1}\right)-f_{2} v_{2}\right] \\
\frac{1}{J_{3}}\left[r_{3}^{2}\left(T_{3}-T_{2}\right)-f_{3} v_{3}\right] \\
\frac{1}{J_{4}}\left[r_{4}^{2}\left(T_{4}-T_{3}\right)-f_{4} v_{4}\right] \\
\frac{1}{J_{4}}\left[-r_{5}^{2} T_{4}-f_{5} v_{5}\right]
\end{array}\right) \\
& v_{t}=\left(\begin{array}{lllll}
v_{1} & v_{2} & v_{3} & v_{4} & v_{5}
\end{array}\right)^{T} \\
& b=\left(\begin{array}{lllll}
r_{1} / J_{1} & r_{2} / J_{2} & r_{3} / J_{3} & r_{4} / J_{4} & r_{5} / J_{5}
\end{array}\right)^{T}
\end{aligned}
$$

$f(\cdot)$ is the function describing the system dynamics, which includes external disturbances and unknown dynamics; $u$ is the control signal; and nominal value for $b$ is assumed to be known. Following the general ADRC design procedure, we first design a second-order extended state observer and then design the ADRC controller.

Rewrite (8) in a state space form as follows:

$$
\left\{\begin{array}{l}
\dot{x}_{1}=x_{2}+b u \\
\dot{x}_{2}=\eta \\
y=x_{1}
\end{array}\right.
$$

with $x_{1}=v_{t}, x_{2}=f(\cdot)$ added as an augmented state and $\eta=\dot{f}(\cdot)$ as unknown disturbance which include the nonlinear system dynamics variations, system model uncertainty, and external disturbances. The reason to increase the system order is to put $f(\cdot)$ as a state such as a state observer can be used to estimate it. Disturbance term $f(\cdot)$ can be estimated by using a nonlinear extended state observer as (10) [20].

$$
\left\{\begin{array}{l}
\dot{z}_{1}=z_{2}+\beta_{1} \mathbf{f a l}\left(y-z_{1}, \alpha_{1}, \delta_{1}\right)+b u \\
\dot{z}_{2}=\beta_{2} \mathbf{f a l}\left(y-z_{1}, \alpha_{2}, \delta_{2}\right)
\end{array}\right.
$$

where, $z_{2}$ is the estimate of the uncertain function $f(\cdot) ; \beta_{1}$ and $\beta_{2}$ are the gains of the observer and then initial setting can be obtained by linearizing $(10)$; fal $\left(e, \alpha_{p}, \delta\right)$ function is defined by (4) and Fig.4. The equations for reference generator can be expressed as (5).

Using the estimation of the total disturbance $z_{2}$, which results from the extended state of observer, the control law is designed as follows:

$$
u(t)=\left[u_{0}(t)-z_{2}(t)\right] \cdot / b
$$

Where $\cdot /$ represents element-by-element division. The control law only depends on partial knowledge of $b$ in (8).

\section{Simulations Results}

Simulations were performed with Matlab-Simulink ${ }^{T M}$ environment for a five-motor winder system. The winder system and the control parameters are given in Appendix. Moreover, all simulations also take into account the dynamics of the torque, flux and current in the motors, in particular with field orientation control law (FOC).

To illustrate the effectiveness of the designed active disturbance rejection control (ADRC) and nonlinear PI (NPI) strategies, a well tuned standard PI controller was also applied to the system for comparison purpose. Note that, the PI, NPI and ADRC controllers were applied on the inner velocities loops, whereas, the PI controllers are applied in the cascaded control structure for current and tension control. All velocity loop controllers use the same load torque compensation terms and the same tension controller. The gains of the PI controller are used for the NPI and ADRC as well.

Moreover, three sets of simulation conditions are used : (i) all parameters are exactly known;

(ii) web stiffness constant is underestimated by a factor of 2 in 

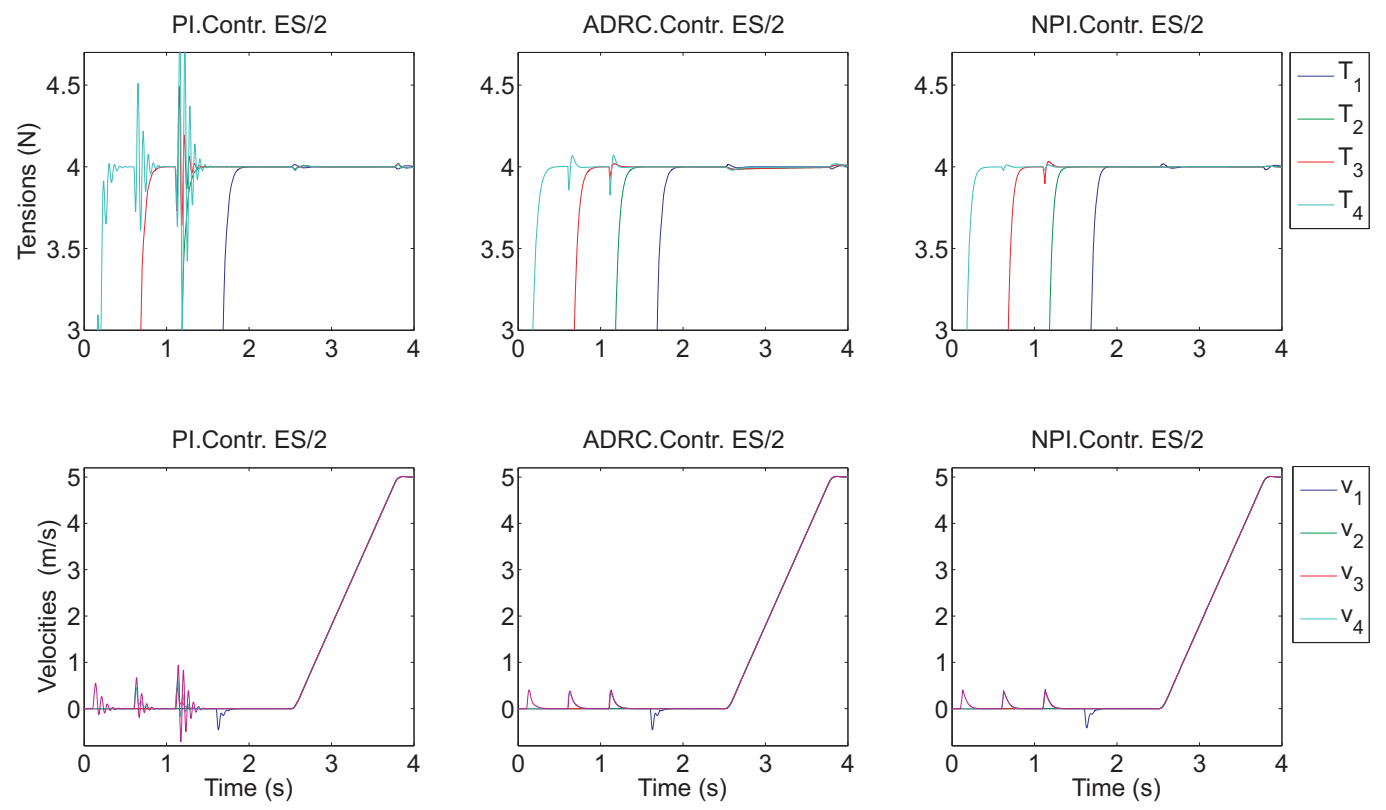

Fig. 7. Tensions and velocities responses for standard PI, NPI and ADRC controllers with underestimation of ES.
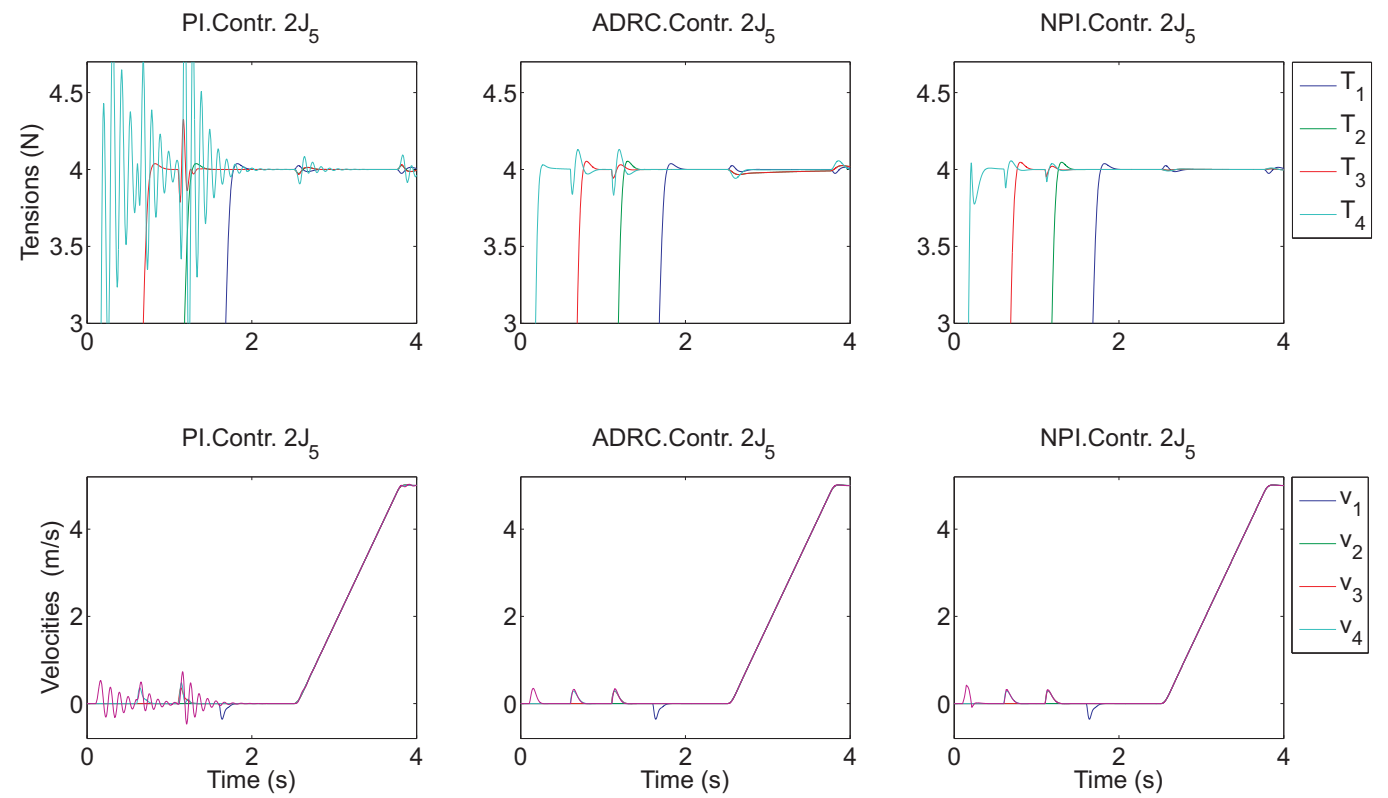

Fig. 8. Tensions and velocities responses for standard PI, NPI and ADRC controllers with overestimation of $J_{5}$.

the controller (identified by $E S / 2$ ), i.e. tension control gains are two times larger than what is called for by the tuning rule; (iii) total inertia $J_{5}$ as seen by motor $M_{5}$ is overestimated by a factor of $2\left(2 J_{5}\right)$.

Step tension reference signals are applied in sequence to the web sections, from the last to the first one, followed by a filtered ramp in speed for the web. Tensions and velocities responses comparisons of standard PI controller, ADRC and nonlinear PI controllers for exact parameters, underestimated of $(E S)$ and overestimation of $\left(J_{5}\right)$ are shown respectively in Fig.6, Fig.7 and Fig.8.
For the nominal parameters, the PI controller responds well to the step input but couplings cause disturbances from the other control loops as seen on Fig.6. The ADRC and NPI exhibit similar step responses but lower sensitivity to disturbances from other control loops. For $(E S / 2)$ and $\left(2 J_{5}\right)$ conditions, we observe that tension and velocity responses to tension reference variations deteriorate significantly, particularly in the case of standard PI controller in Fig.7 and Fig. 8 respectively. These variations can be seen from the larger oscillations during the transient responses because of tension and velocity coupling (left of figures). However, the 
TABLE I

PERFORMANCE INDICE (ISE) OF TENSION ERRORS $\triangle T_{3}$ AND $\triangle T_{4}$ FOR EXACT AND UNDERESTIMATED PARAMETERS $E S$

\begin{tabular}{|l||cc||cc||cc|}
\hline \multirow{2}{*}{ Controller type } & \multicolumn{2}{|c||}{ PI standard } & \multicolumn{2}{c||}{ ADRC } & \multicolumn{2}{c|}{ NPI } \\
\cline { 2 - 7 } & $\triangle T_{3}$ & $\triangle T_{4}$ & $\triangle T_{3}$ & $\triangle T_{4}$ & $\triangle T_{3}$ & $\triangle T_{4}$ \\
\hline Exact parameters & 3.18 & 6.30 & 0.27 & 0.45 & 0.16 & 0.01 \\
\hline$E S / 2$ parameter & 7.07 & 34.3 & 0.77 & 1.03 & 0.43 & 0.20 \\
\hline $2 J_{5}$ parameter & 3.18 & 146 & 0.27 & 1.83 & 0.17 & 1.08 \\
\hline
\end{tabular}

NPI and ADRC controllers provide higher control quality of the web tensions and velocities. The improvement appears as a significantly increased damping of the oscillations in the response and a shorter settling time after the disturbance. Moreover, the system exhibits much stronger robustness with respect to inertia winder and elasticity modulus variations of the web during the winding process.

To compare quantitatively the responses of the controllers, the integral of the square of the error (ISE) performance index is used for exact parameters and for $(E S)$ underestimated by $50 \%$ and $\left(J_{5}\right)$ overestimated by $100 \%$ respectively. The value of ISE indices, evaluated over $t=0 s$ to $4 s$, for tension errors $\triangle T_{3}$ and $\triangle T_{4}$ are given in Table I . These results reveal that the NPI controller performs the best and that the proposed NPI and ADRC controllers have better performance in tension control over the PI controller and offer good robustness to variations of inertia and elasticity modulus.

\section{CONCLUSION}

In winding system, disturbance rejection is an efficient mean to ensure higher quality processing. Simulation results show that the performance and disturbance rejection with ADRC and nonlinear PI controllers is significantly improved as compared to a system with standard PI controllers. Part of the design procedure for ADRC and nonlinear PI controllers is empirical but linear analysis allows to determine initial settings for the gains. In future work, we are planning the design of the NPI and ADRC controller with an optimization process.

\section{APPENDIX}

TABLE II

WINDER SYSTEM PARAMETERS

\begin{tabular}{|l||l||l|}
\hline Elasticity modulus of the web & $E$ & $1.6 \cdot 10^{8} \mathrm{Nm}^{2}$ \\
\hline Section of the web & $S$ & $2.75 \cdot 10^{-5} \mathrm{~m}^{2}$ \\
\hline Web length between two rolls & $L_{1}=\cdots=L_{4}$ & $2 \mathrm{~m}$ \\
\hline Radius of roll k & $r_{1}=r_{5}$ & $0.5 \mathrm{~m}$ \\
& $r_{2}=r_{3}=r_{4}$ & $0.25 \mathrm{~m}$ \\
\hline Inertia moment of roll k & $J_{1}=J_{5}$ & $0.6 \mathrm{kgm}^{2}$ \\
& $J_{2}=J_{3}=J_{4}$ & $0.15 \mathrm{kgm}^{2}$ \\
\hline Viscous friction of motor $\mathrm{k}$ & $f_{1}=\cdots=f_{5}$ & $0.04 \mathrm{Nm} \frac{\mathrm{s}}{\mathrm{rad}}$ \\
\hline Web tension reference & $T_{k_{\text {ref }}, k=1, \ldots, 4}$ & $4 \mathrm{~N}$ \\
\hline Web master velocity reference & $v_{2_{\text {ref }}}$ & $5 \mathrm{~m} / \mathrm{s}$ \\
\hline
\end{tabular}

TABLE III

Control Parameters

\begin{tabular}{|l||l|}
\hline PI controller & $\begin{array}{l}\text { Tension }: k_{i}=2.75 \cdot 10^{3}, k_{p}=170.66, \\
\text { Velocity }: k_{i_{1,5}}=1560, k_{i_{2}}=62.5, k_{i_{3,4}}=390.6 \\
\end{array}$ \\
\hline ADRC controller & $k_{p_{1,5}}=31.23 \cdot 10^{3}, k_{p_{2}}=3.1, k_{p_{3,4}}=7.79$ \\
\hline NPI controller & $R_{1,2}=2 \cdot 10^{3}, \delta_{1,2}=0.1, \alpha_{1,2}=0.7$ \\
\hline
\end{tabular}

\section{REFERENCES}

[1] K.N. Reid, K.H. Shin, and K. C. Lin, "Variable-gain control of longitudinal tension in a web transport system," AMD, vol. 149, Web Handling, ASME, 1992, pp. 87-100.

[2] P. Lin and M.S. Lan, "Effects Of PID gains for controller with dancer mechanism on web tension," Proc. of the Second Inter. Conf. on Web Handling, Stillwater, Oklahoma, 1993, pp. 66-76.

[3] B.T. Boulter, "The Effect of speed loop bandwidths and line-speed on system natural frequencies in multi-span strip processing systems," IEEE IAS Annual Meeting, Aug. 1997, vol.3, pp. 2157-2164.

[4] H. Koç, D. Knittel, M.D. Mathelin, and G. Abba, "Robust gain-scheduled control of winding systems," IEEE Conf. Decision and Control, Sidney, Australia, Dec. 2000. vol. 4, pp. 4116-4119.

[5] E. Laroche, H. Koç, D. Knittel, and M.D. Mathelin, "Web winding system robustness analysis via $\mu$-analysis," Proc. 10th IEEE Inter. Conf. on Control Applications, Sep. 2001, pp. 948-953.

[6] H. Koç, D. Knittel, M. Mathelin, and G. Abba, "Modeling and robust control of winding systems for elastic webs," IEEE Trans. on Control System Technology, 2002. vol. 10, No. 2, pp. 197-208.

[7] S. Jee, S. Kim and K.H. Shin, "Adaptive fuzzy control of tension variations due to the eccentric unwinding roll in multi-span web transport systems," Proceedings of ASME Dynamic System and Control Division, 1999, vol. 67, pp. 877-882.

[8] C. Wang, and Y.Z. Wang, "Research on precision tension control system based on neural network," IEEE Transaction on Industrial Electronics, 2004, vol. 51, No. 2, pp. 381-386.

[9] A. Angermann, M. Aicher and D. Schroder, "Time-optimal tension control for processing plants with continuous moving webs," Proc. 35th Annual Meeting IEEE Industry Applications Society, Rome, Oct. 1999, vol. 5, pp. 3505-3511.

[10] P.R. Pagilla and all "Dynamics and control of accumulators in continuous strip processing lines," IEEE Transactions on Industry Applications, 2001, vol. 37, pp. 934-940.

[11] M.D. Baumgart, and L.Y. Pao, "Robust Lyapunov-based feedback control of nonlinear web-winding systems," Proceedings of IEEE Conference on Decision and Control, Jun. 2003, vol. 6, pp. 6398-6405.

[12] D. Sun, "Comments on Active Disturbance Rejection Approach," IEEE, Trans. On Industrial Electronics, 2007, vol. 54, No. 6, pp. 3428-3429.

[13] J. Han, "From PID to Active Disturbance Rejection Control," IEEE, Trans. On Industrial Electronics, 2009, vol. 56, No. 3, pp. 900-906.

[14] F. Mokhtari, P. Sicard and A. Hazzab, "Cascade decentralized nonlinear PI control continuous production process," ELECTRIMACS, Québec, Canada, Jun. 2008, CD-ROM.

[15] T. Sakamoto, "Decentralized controller design of web tension control system in terms of interactions," ISIE, 1999, Bled, Slovenia, pp. 14661471.

[16] P.R. Pagilla, N.B. Siraskar, and R.V. Dwivedula, "Decentralized control of web processing lines," IEEE Transaction on control systems technology, Jan. 2007, vol. 15, No. 1, pp. 106-117.

[17] N.I. Giannoccaro, T. Sakamoto, "Importance of overlapping decomposition for a web tension control system," Advances in Production Engineering and Management (APEM) Journal, 2007, 2(3), pp. 135-145.

[18] D. Knittel, D. Gigan, "Robust decentralized overlapping control of large scale winding system," Proc. American Control Conference, 2002, vol. 3, pp. $1805-1810$.

[19] W. Zhou, S. Shao and Z. Gao, "A stability study of the active disturbance rejection control problem by a singular perturbation approach," Applied Mathematical Sciences, 2009, vol. 3, no. 10, pp. 491-508.

[20] J. Han, "A class of extended state observers for uncertain systems," Control and Decision, 1995, vol. 10, No. 1, pp. 85-88. 